\title{
Sistematizaciones de competencias de los profesionales de la información. Valoración de la relación de Decidoc por los asociados de Sedic
}

\author{
Por Carlos Tejada Artigas y Luis Rodríguez Yunta
}

\begin{abstract}
Resumen: Se recopilan las sistematizaciones más importantes realizadas sobre las competencias y aptitudes necesarias para el desarrollo profesional en información y documentación. La formalización de las competencias aporta diferentes ventajas a la profesión: contribuye a su afianzamiento en el mercado laboral definiendo perfiles y supone un instrumento fundamental para poder orientar la formación tanto de base como continua. Finalmente, se somete a valoración de los socios de Sedic la relación de eurocompetencias del proyecto Decidoc, el cuadro que mejor se adecua a las características de los profesionales europeos y españoles.
\end{abstract}

Palabras clave: Profesionales de la documentación, Competencias profesionales, Encuestas, Decidoc, Sedic.

\begin{abstract}
Luis Rodríguez Yunta, licenciado en historia por la Universidad Complutense de Madrid. Experiencia profesional en el Centro de Información y Documentación Científica (Cindoc) del Csic: desde 1986 a 1989 en el Servicio de Acceso al Documento Primario y desde 1990 en el Departamento de Documentación y Análisis Bibliométrico en Ciencias Humanas. Ha impartido diferentes cursos. Miembro de la Junta Directiva de Sedic.
\end{abstract}

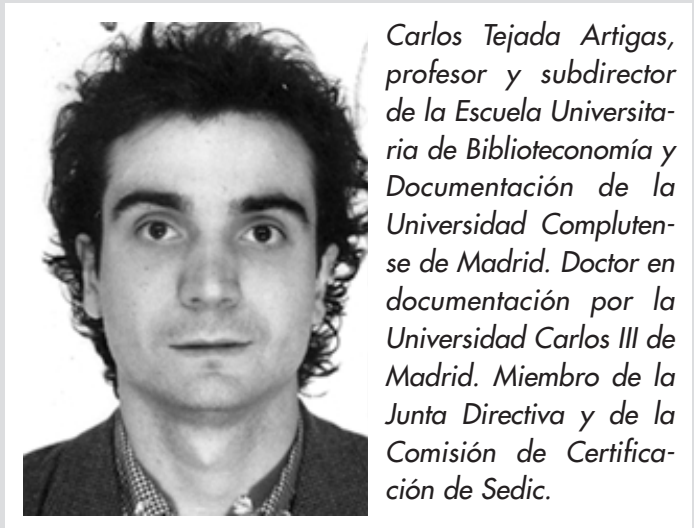

Title: Systematising the competencies of information professionals: an assessment of the Decidoc set of competencies performed by the members of Sedic.

Abstract: The article compiles data on the principal work performed on systematising the competencies and skills needed for professional development within the field of library and information science. Setting up competencies provides several benefits to the profession: by helping to secure its position within the job market through the definition of profiles and by serving as a basic tool for orienting both formal and continuing education. The article concludes with an evaluation -performed by members of the professional association Sedic (Sociedad Española de Información y Documentación Científica) - of the European Reference Index of Competencies (developed within the Decidoc project), which lists the main characteristics expected of European and Spanish professionals.

Keywords: Library and information professionals, Professional competencies, Surveys, Decidoc, Sedic (Sociedad Española de Documentación e Información Científica).

Tejada Artigas, Carlos; Rodríguez Yunta, Luis. "Sistematizaciones de competencias de los profesionales de la información. Valoración de la relación de Decidoc por los asociados de Sedic". En: El profesional de la información, 2001, enero-febrero, v. 12, n. 1, pp. 10-17.

\section{La competencia. Definición y utilidad}

Se pueden encontrar diferentes definiciones de este concepto en relación con la acción en un puesto de trabajo. En un nivel general, Bung la considera como: "el conjunto de conocimientos, destrezas y aptitudes cuya finalidad es la realización de actividades definidas y vinculadas a una determinada profesión" (Rial, 2000). En nuestro sector, la Relación de eurocompe- tencias en información y documentación (Reid) define las competencias de una manera similar: "se entienden como el conjunto de capacidades necesarias para el ejercicio de una actividad profesional y el dominio de los comportamientos correspondientes" (Reid, 2000, p. 11).

La Special Library Association en sus Competencies for special librarians for 21st century (Spiegel- 
man, 1997) considera también que en su definición entran los conocimientos y las aptitudes. Así, determina que se necesitan 2 tipos de competencias: las profesionales y las personales. Las primeras abarcarían los conocimientos y la habilidad para utilizarlos como base para proporcionar los distintos servicios; mientras que las personales consisten en "las habilidades, actitudes y valores que le permiten a los bibliotecarios trabajar de forma eficiente; ser buenos comunicadores; enfocarse en el aprendizaje continuo durante toda su carrera; demostrar el valor agregado de sus contribuciones; y sobrevivir en el nuevo mundo laboral" (Reid, 2000, p. 10).

Uno de los grandes defensores de la formalización de las competencias, Jean Meyriat (2001), resalta la importancia de las aptitudes. Defiende que los conocimientos carecen de sentido sin ellas, ya que la competencia, además de ser un recurso, es un proceso en el que cada individuo de una forma distinta pone en acción sus conocimientos. En esta línea, la Reid precisa que las aptitudes son "las disposiciones, naturales o adquiridas, que provocan una manera de comportarse" (p. 14).

\section{«Para una eficaz economía del conocimiento en una institu- ción se necesita que todos los empleados tengan habilidades de alfabetización en la gestión de la información» (Tfpl)}

Las competencias y aptitudes identificadas en las diferentes sistematizaciones provienen de distintas áreas de conocimiento. Así, Maurice B. Line (1998) defiende que los bibliotecarios deben tener un conjunto de competencias pertenecientes a distintas disciplinas, lo que produce una cierta diversificación de la profesión: "está suficientemente claro que los bibliotecarios hoy tienen que tener un conjunto de conocimientos y saberes de diferentes dominios que estén aplicados a una actividad particular, pero que, todos ellos, no constituyen un corpus que tengan lugar en el seno de una única disciplina. Sólo una pequeña (pero importante) parte concierne específicamente a la biblioteconomía" (Line, 1998, pp. 45-46). Esta misma idea es recogida por Dominique Arot (2000) quien va incluso más lejos al afirmar que las competencias ligadas a la comunicación, a la pedagogía y a la formación tienen un papel más importante que las relacionadas con la gestión de las colecciones.

La sistematización de las competencias es útil para los diferentes integrantes de la profesión. Así, a los profesionales les faculta para definir su propio perfil y señalar sus puntos fuertes y débiles ante el mercado la- boral; a los futuros profesionales les permite percibir los objetivos de su formación; a los propios docentes para que dirijan sus enseñanzas; y para los empleadores, a fin de poder evaluar los diferentes currícula y definir sus necesidades de personal.

Bertrand Calenge (1995) determina en qué aspectos de nuestra profesión puede proporcionar ventajas la formulación de competencias:

—autoevaluación sobre la propia formación,

—en la elaboración y reforma de los planes de estudio,

—en el diseño de programas de formación continua,

—en un posible sistema de certificación, y

—en la asignación de tareas en un ambiente de trabajo.

Tal como señala Meyriat: "nuestra profesión tiene su propia responsabilidad para definir y regular sus propias normas" (2001, p. 1). La sociedad de la información está provocando que los límites entre las profesiones se estén desdibujando, lo que conlleva que debamos tener claras nuestras competencias para así poder defender nuestros espacios de actuación.

\section{Proyectos de elaboración de cuadros de competencias en información y documentación}

Es abundante la bibliografía que ha tratado de sistematizar las funciones que debe cumplir el profesional de la información y documentación. Las asociaciones profesionales y también otros tipos de instituciones relacionadas con este sector, así como diferentes estudiosos, han elaborado cuadros y relaciones que identifican y sistematizan las competencias. A continuación se señalan los trabajos que, a nuestro juicio, consideramos más significativos.

2.1. Competencias para bibliotecarios especializados de la Special Library Association (SLA). La SLA elaboró las Competencias para bibliotecarios especializados del siglo XXI (Spiegelman, 1997) que, como ya señalamos, se dividen en 2 grupos: profesionales y personales. Produjeron un amplio debate en la profesión, tal como recoge la bibliografía y las listas de discusión electrónicas; así por ejemplo, Graerly y Greenman (1998) destacan que no sólo son útiles en las bibliotecas especializadas, sino que son adaptables a otros tipos de centros. En esta línea, Pamela Scott y Anne Kirby (1999) analizaron su integración en los ambientes de trabajo a partir de entrevistas con diferentes profesionales de la información. 
Hay que destacar también el estudio de Sheila Webber (1999), que las compara con las del Consejo de Europa y con las de la consultora inglesa Tfpl. Para esta autora las competencias de la SLA se acercan más a las realidades prácticas que el resto.

Wrigth (1997) defendió que el documento de la SLA sirviera como referente para los formadores. La misma SLA, Alise (Association for Library and Information Science Education) y MLA (Medical Library Association) (1998) evaluaron de qué manera los planes de estudio en información y documentación habían adoptado estas competencias.

\subsection{Recomendaciones del Consejo de Europa so-} bre perfiles y competencias de los profesionales de la información y trabajadores del conocimiento que trabajan en las industrias e instituciones culturales. Su objetivo es "mantener elementos importantes de una política cultural orientada a las nuevas tecnologías para animar el desarrollo de las instituciones e industrias culturales asegurando que sus profesionales de la información y proveedores de contenidos estén listos para participar totalmente en la nueva cadena de la información" (Council of Europe, 1998).

El documento distingue entre profesionales de la información y trabajadores del conocimiento. Los primeros son los mediadores entre creadores, proveedores, usuarios y las tecnologías de la información. Tienen competencias tecnológicas para organizar y recuperar información, así como competencias profesionales y sociales para procesarla según las necesidades de los usuarios. Por su parte, los trabajadores del conocimiento facilitan además un valor añadido a la información, por lo que se les considera "analistas simbólicos", capaces de desarrollar nuevos servicios y productos basados en el conocimiento.

Los nuevos perfiles se agrupan en 4 áreas: diseño y tecnología, gestión y tecnología, contenido y tecnología, y distribución y tecnología. En esta última es donde mejor encajaría nuestra profesión: broker de la información como librero o bibliotecario multimedia, webmaster, archivero/documentalista de productos electrónicos y tele-tutor.

El Consejo de Europa aboga por profesionales de la información y trabajadores del conocimiento que asuman una serie de objetivos:

- Adquirir y procesar información para mediar y realizar productos de información de mercado.

- Ser conscientes de las características, potenciales y límites de los medios de comunicación digitales.

- Conocer las necesidades y requisitos de los distintos grupos de usuarios, el profesional y el privado.
- Ser capaces de transferir el desarrollo cultural y social al proceso de la producción y difusión de información.

- Tener actitudes de trabajo orientadas al servicio y al mercado.

- Poder evaluar los medios de comunicación, los servicios de información y los productos, teniendo en cuenta los tipos de información, los requisitos del usuario y los mercados.

Las competencias necesarias para todo ello se dividen en 8 grupos: habilidades directivas, organizativas, capacidades creativas, competencias en informática, actitudes personales, habilidades legales, de comunicación y lingüísticas.

2.3. Habilidades para la gestión del conocimiento. Un estudio de Tfpl y de la Comisión de Biblioteca e Información del gobierno del Reino Unido. El informe Skills for knowledge management (Tfpl, 1999) fue realizado por la consultora Tfpl y por la Library and Information Commission del Reino Unido. Se distinguen las habilidades requeridas por todos los empleados de la organización y las que necesitan los diferentes perfiles de profesionales de la gestión del conocimiento. Para una eficaz economía del conocimiento en una institución se necesita que todos los empleados tengan habilidades de alfabetización en la gestión de la información.

\section{«La formalización de compe- tencias es fundamental para poder diseñar con efectividad los programas de formación»}

Por lo que respecta a los perfiles en gestión del conocimiento diferencia 3 tipos: competencias básicas, habilidades de supervivencia y habilidades específicas de la gestión del conocimiento. Las primeras están compuestas por el bagaje educativo, profesional y técnico del candidato, más su experiencia y desarrollo. Las segundas son las requeridas en cualquier puesto para que se puedan aplicar eficazmente las competencias básicas: habilidades de comunicación, gestión de proyectos, trabajo en equipo y capacidades comerciales entre otras. Las habilidades específicas de la gestión del conocimiento son una mezcla de habilidades de gestión del cambio y de gestión de la información.

2.4. Criteria for information science del Institute of Information Scientists. En este informe, el IIS, del Reino Unido, identificó las habilidades necesarias para los especialistas de información, dividiendo las competencias en 4 secciones: 
-Área básica de ciencias de la información: características de la información, sus proveedores y usuarios, fuentes, recuperación y almacenamiento, análisis y teoría de las ciencias de la información.

- Gestión de la información: planificación, comunicación, sistemas de gestión y control de la información, gestión de recursos humanos, gestión financiera, promoción, economía, marketing y factores políticos, éticos, sociales y legales.

- Tecnología de la información: sistemas informáticos, hardware y software, telecomunicaciones, aplicaciones de tecnología de la información y cuestiones del entorno.

- Habilidades auxiliares: técnicas de investigación, habilidades lingüísticas e idiomas.

2.5. Proyecto Futureprof. Pretendía identificar las ocupaciones con un futuro más prometedor por estar basadas en el desarrollo de la sociedad de la información y en las nuevas tecnologías (Euro-Chambres, 1999). Se identificaron 6 perfiles profesionales: broker de la información, nuevo diseñador de medios de comunicación, nuevo técnico de medios de comunicación, técnico de la Red, especialista de marketing electrónico y experto en nuevas tecnologías (IT pilot).

2.6. Relación de eurocompetencias en información y documentación. El proyecto Decidoc (Desarrollar las Eurocompetencias en Información y Documentación). Fue realizado en el marco del programa europeo Leonardo da Vinci y tenía como objetivo más importante "promover la cooperación entre todos los socios [del proyecto] para conseguir un mejor conocimiento de las competencias en el campo profesional de la información y documentación y una mayor transparencia de las competencias clave adaptadas al desarrollo tecnológico, a la competitividad de las empresas y a las necesidades del mercado de trabajo" (Commission Européenne, 2001, p. 5).

El proyecto, financiado por la Comisión Europea, fue llevado a cabo por asociaciones europeas de profesionales integradas en Ecia (Abd-Bvd, Adbs, Aslib, $D g i$, Incite y Sedic). Además se sumaron las asociaciones profesionales de otros 3 países europeos: Info. Doc. Rom de Rumania, ASA de Suiza y Skip de la República Checa.

Jean Meyriat justificaba el entorno europeo para definir las competencias en información y documentación de la siguiente manera: "por dos razones principales, la primera es que el reconocimiento similar en todos los países de una misma profesión da más peso, más fuerza a esta profesión en su relación con las autoridades públicas, con los gobiernos, con la opinión pública o con los empleadores. La segunda razón es que el reconocimiento en muchos países puede ayudar a la movilidad profesional de los profesionales entre las fronteras; por esta razón decidimos hacer este trabajo común y buscar el interés de la Comisión Europea" (Meyriat, 2001, p. 1).

El principal logro de Decidoc fue la Relación de eurocompetencias en información y documentación (Reid) que tuvo como objetivo ser "un instrumento de referencia aceptado en el marco europeo, que permita la evaluación objetiva de las competencias de los profesionales de la información y documentación" (Commission Européenne, 2001, p. 14).

\section{«En un entorno de cambio, en el que las distintas profesiones relacionadas con la sociedad de la información van desdibu- jando sus límites, hay que po- der sistematizar los ámbitos profesionales"}

En esta sistematización se identifican 30 campos, divididos en 4 grupos, en los que se puede ejercer. Los 10 primeros se podrían definir, en palabras de Meyriat, como "el corazón de la profesión" (Meyriat, 2001, p. 3), son los conocimientos teóricos y prácticos fundamentales. Aquí encontramos las competencias "clásicas" de la profesión: identificación y selección de las fuentes de información, tratamiento documental, búsquedas documentales o los servicios de difusión. El grupo segundo incluye la comunicación; el tercero la gestión de la organización y el cuarto y último los conocimientos externos a nuestro campo.

En cada competencia se determinan 4 niveles sucesivos que se corresponderían con los 4 niveles de objetivos pedagógicos que distinguen los especialistas en formación. El más básico es el de "sensibilización": se conoce la existencia de los elementos de una determinada función y se maneja el vocabulario para identificar los problemas. El segundo, "conocimiento de las prácticas", es en realidad el primer nivel profesional ya que el sujeto puede manejar herramientas básicas y es capaz de efectuar ciertos trabajos técnicos. En el tercero, "dominio de herramientas", ya se controlan las distintas técnicas y por ello se está capacitado para el desarrollo de nuevas herramientas. El nivel superior, "dominio metodológico", supone la capacidad para planificar estratégicamente y concebir herramientas y productos nuevos. En cada una de las 120 divisiones que resultan se señala una serie de tareas como ejemplo de lo que sabe hacer la persona que se encuentra en cada nivel. 
Además se establecen las 15 aptitudes básicas de la profesión. Muchas de ellas no son privativas de nuestro campo, pero su "importancia puede ser decisiva en el perfil de un buen profesional de la idoc" (Reid, 2000, p. 14).

2.7. Propuestas realizadas desde el ámbito universitario. Como ya hemos señalado, la formalización de competencias es fundamental para poder diseñar con efectividad los programas de formación. Así, desde el ámbito universitario también se han elaborado diferentes propuestas de las que señalamos tan sólo algunas de ellas.

El IV Encuentro de directores de escuelas de bibliotecología y ciencia de la información del Mercosur (Valentim, 2000) determinó una sistematización de las competencias que debía tener un graduado en estas disciplinas de una universidad del Mercosur. Se diferenciaban competencias en comunicación y expresión, técnico-científicas, gerenciales, y sociales y políticas.

\section{«Nuestra profesión sigue sin tener un reconocimiento social óptimo, por lo que la definición por medio de competencias es doblemente valiosa»}

Noel Angulo Marcial (1999), a partir de una revisión bibliográfica, propuso un conjunto de 10 habilidades informativas para orientar la formación de las instituciones de educación superior, cada una de las cuáles se desglosa en una serie de especificaciones.

Un estudio elaborado en la École Supérieure d'Information Documentaire de Ginebra (Deschamps, 1999) reafirmó la importancia de las aptitudes. Es más, señalaba que las competencias "transversales" no necesariamente están ligadas a la técnica documental.

En España, la profesora de la Universidad de Barcelona, Montserrat Sebastià (1996), resaltó la importancia de las competencias tecnológicas, e incluso denominó al profesional de la documentación como "infotecnólogo".

\section{Valoración efectuada por los socios de Sedic sobre las competencias}

3.1. Compromiso de Sedic con el desarrollo profesional. El asociacionismo juega un papel relevante para la consolidación de la documentación como un sector profesional. Consciente de ello, la Sociedad Española de Documentación e Información Científica (Sedic) pretende ofrecer un apoyo para la carrera profesional a través de la formación continua, de la certificación profesional y de diferentes acciones referentes al empleo. Sedic tiene desde el año 1994 un compromiso firme con la certificación de profesionales, que se ha manifestado en la creación de un sistema de certificación y en la participación activa en los proyectos europeos Decidoc y Certidoc (Portela, 1997; Fernández, 2002).

En la actualidad su Servicio de Certificación de Profesionales en Información y Documentación certifica a profesionales en 4 niveles y utiliza como instrumento para la verificación del perfil profesional la relación de competencias del proyecto Decidoc, antes expuesta. El proyecto Certidoc, aprobado por la Comisión Europea recientemente, pretende elaborar un sistema europeo de certificación de competencias profesionales en materia de información y documentación.

En cuanto al empleo, se creó un grupo de trabajo específico, con el nombre de Activa, que se plantea entre otros objetivos la potenciación de la bolsa de empleo de Sedic y la realización de actividades de investigación y de orientación sobre el mercado laboral.

3.2. Estudio realizado sobre la situación profesional de los socios de Sedic. Una de las acciones de Activa ha sido la acometida de un estudio (Tejada; Rodríguez, 2002) para conocer el desarrollo profesional y la situación laboral de los socios con una doble finalidad: una académica, a fin de tener una visión completa de este importante colectivo profesional; y una segunda más de gestión, ya que las diversas opiniones de este colectivo pueden ayudar a la planificación de diferentes actividades de esta asociación. Para ello se establecieron unos objetivos específicos entre los que figuraba la valoración de las competencias y actitudes que necesita un profesional de la información.

El estudio se basó en la realización de 2 cuestionarios que se enviaron por correo postal a la totalidad de los socios de Sedic. El primero de ellos, más genérico, se dividía en 4 apartados:

a) Formación, donde aparecían las preguntas sobre la formación tanto académica como continua de los socios y sobre la opinión que tienen acerca de los cursos de Sedic.

b) Empleo, agrupaba diferentes cuestiones sobre el trabajo en información y documentación que desarrolla cada asociado en la actualidad.

c) Desarrollo profesional, se ocupaba de la valoración de las competencias y actitudes de Decidoc, así como de la satisfacción y reconocimiento profesional de los socios.

d) Datos personales, concentraba algunas preguntas para conocer información sociodemográfica. 
El segundo cuestionario, menos extenso, iba dirigido tan sólo a los socios inscritos en la bolsa de trabajo y recogió sus opiniones sobre la misma, el grupo Acti$v a$ y las formas en que este colectivo busca trabajo.

Para el diseño de estos estudios se analizaron diferentes encuestas ya realizadas para medir aspectos formativos y laborales de los profesionales de la información y documentación. Así, por ejemplo, se analizó especialmente el cuestionario que realiza desde el año 1964 la Adbs francesa a sus asociados, por la similitud de sus objetivos (Roederer, 2000).

Los cuestionarios se mandaron a los socios en 2 ocasiones aprovechando los envíos de información de Sedic. El primero se realizó en junio de 2001 y el segundo en septiembre de ese mismo año. Para facilitar la respuesta, se abrió un apartado postal y se adjuntó un sobre de respuesta ya franqueado.

El cuestionario general fue contestado por 326 socios (el 29\% de los 1.122). El específico de la bolsa de empleo fue rellenado por $149(74,5 \%)$ —l número de inscritos en la bolsa de empleo era de 200-. Para el procesamiento de los datos obtenidos se utilizó el soft- ware estadístico Spss 10.0, que permitió diferentes cruces de variables.

\subsection{Valoración de las competencias por los so-} cios de Sedic. Se tuvieron en cuenta las competencias del proyecto Decidoc puesto que la propia Sedic, como ya hemos señalado, participó en este proyecto, y por entender que es la sistematización que más se acerca a la situación española. La valoración se realizó por medio de una puntuación del 1 al 5 , y la alta puntuación media demostró la pertinencia de este documento.

La competencia más valorada fue la búsqueda de información (4,71 puntos), lo cual se corresponde con el hecho de ser la tarea más realizada por los socios en las unidades de información como reflejaba el análisis de otra pregunta realizada en el cuestionario (Tejada; Rodríguez, 2002, pp. 35-36). La importancia de las consultas en el quehacer diario del profesional de la información también ha sido señalado por otros estudios $(F I D, 1997)$. Este dato nos permite afirmar que el fenómeno de la desintermediación del que algunos autores (Gellman, 1996; Noble, 1998) habían alertado, no

Gráfico 1. Valoración por los socios de Sedic de las competencias del proyecto Decidoc. Base: socios de Sedic que contestaron al cuestionario.

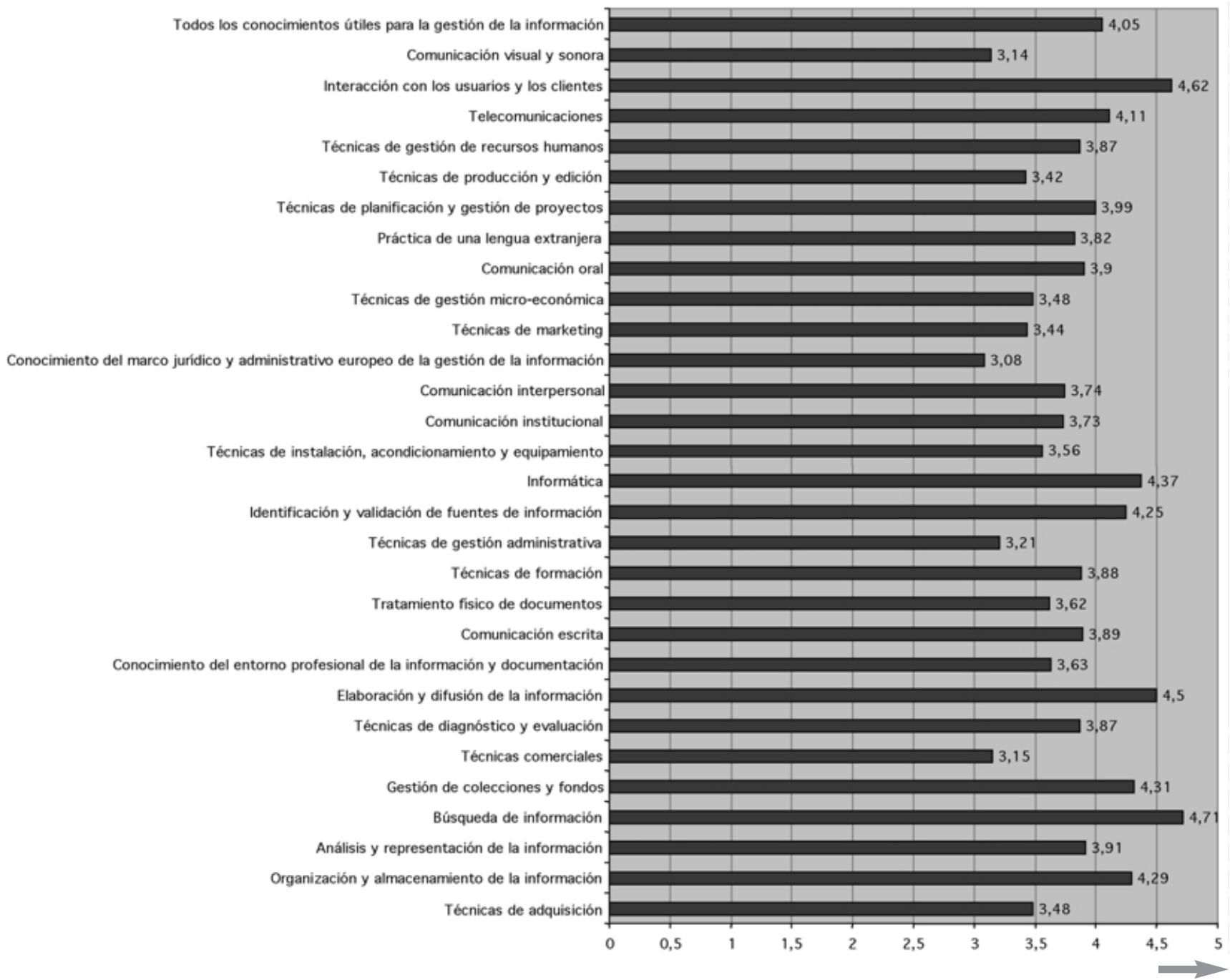


ha cambiado substancialmente el papel del profesional como mediador.

También queda reflejado en la encuesta el sentido de servicio de la profesión, señalado por diversos autores (Carbo, 1999; Gorman, 1999), ya que la segunda competencia más valorada ha sido la interacción con los usuarios y los clientes (4,62). El tercer puesto fue ocupado por la elaboración y difusión de la información $(4,50)$. Este valor de los contenidos es también apuntado por la bibliografía especializada (Sturges, 1999; Carbo, 1983).

Otra característica que se puede observar de la lectura de los resultados es la diversificación de la profesión ya que las 30 competencias evaluadas han tenido una puntuación positiva. La más negativa ha sido un 3,08, lo que indica cómo el profesional de la documentación hoy en día debe tener un amplio abanico de capacidades (en el gráfico 1 se presentan estos datos). También queda reflejada en los resultados de la encuesta la adecuación de las aptitudes identificadas en el proyecto Decidoc, ya que todas ellas son muy valoradas, tal y como muestra el gráfico 2 .

\section{Conclusiones}

En un entorno de cambio, en el que las distintas profesiones relacionadas con la sociedad de la información van desdibujando sus límites, es fundamental poder sistematizar los ámbitos profesionales. Además, nuestra profesión sigue sin tener un reconocimiento social óptimo, por lo que la definición por competencias es doblemente valiosa. Esta formalización no sólo es útil en la satisfacción profesional, sino que además es una potente herramienta para dirigir la formación tanto de base como continua y como apoyo en el área de recursos humanos de las organizaciones.

Las diferentes relaciones y sistematizaciones de competencias recogen la amplitud del abanico de conocimientos y aptitudes necesarios para el desarrollo profesional. Estas competencias no sólo proceden de la biblioteconomía y documentación sino que también provienen de otros campos comunes a las profesiones relacionadas con la nueva sociedad de la información y del conocimiento.

El proyecto Decidoc es el referente más cercano y su adecuación al desarrollo de los profesionales españoles se ve confirmado a través de la opinión de los socios de Sedic, ya que cada una de las competencias fue valorada como muy determinante.

\section{Bibliografía}

Arot, D. "Les valeurs professionnelles du bibliothécaire". En: Bulletin des bibliothèques de France, 2000, v. 45, n. 1, pp. 33-41. Consultado en: 06-11-02.

http://www.enssib.fr/bbfl

L'Association des Professionnels de l'Information et de la Documentation. Référentiel des métiers-types et compétences des professionnels de l'information et documentation. Paris: Adbs, 1998. Isbn 2-84365-010-0.

Bérard, R. "L'évaluation des compétences et des personnels". En: Bulletin des bibliothèques de France, 2000, v. 45, n. 1, pp. 62-70. Consultado el: 06-11-02.

http://www.enssib.fr/bbfl

Calenge, B. "Peut-on définir la bibliothéconomie?". Bulletin des bibliothèques de France, 1998, v. 43, n. 2, pp. 8-24.

Calenge, B. "Formation et recherche bibliothéconomiques en France: lecture d'une complexité".

http://www.bibliothek-saur.de/2000_1/18-26.pdf. Consultado el: 6-12-02.

Carbo, T. "The changing role of the information professional and its implications for library and information service education". En: Boehm, E. H.; Buckland, M. K. (eds.) Education for information management: directions for the future. Santa Barbara: International Academy, 1983, pp. 19-22.

Carbo, T. "The librarian within the large family of information professions: an American perspective". En: FID review, 1999, v. 1, n. 1, pp. 2427.

Commission Européenne. Direction Général XXII. Project Decidoc. Rapport final. Bruxelles, 2001.

Cosandier, J. F. Le project européen Decidoc et le développement de systèmes nationaux de certification dans le domaine $I+D, 1999$. Consultado el: 17-05-00.

http://www.svd-asd.org/france/DossierF.html 
Council of Europe. Draft recommendation n. R (98). On cultural work within the information society: new professional profiles and competencies for information professionals and knowledge workers operating in cultural industries and institutions. Strasbourg, 1998. CC-Cult (98) 21.

Council of Europe. New professional profiles and competencies for information professionals and knowledge workers operating in cultural industries and institutions: results of the consultation process (December 1998 February 1999). Strasbourg, 1999. Consultado el: 17-08-00.

http://culture.coe.fr/books/eng/ecubook\%20f.1.htm

Dalbin, S.; Meyriat, J. "Utiliser le référentiel des compétences et emplois de l'Adbs. Un exemple: redéfinir un poste de travail". En: Documentaliste-sciences de l'information, 1996, v. 33, n. 4-5, pp. 244-247.

Dalbin, S.; Sutter, E. "Utiliser le référentiel des compétences et emplois de l'Adbs. Un exemple: caractériser ses sompétences pour évoluer vers d'autres emplois". En: Documentaliste-sciences de l'information, 1997, v. 34, n. 1 , pp. 29-32.

Deschamps, J. Nouvelles competences en information documentaire et technologie de l'information: etude des offres d'emploi du Bulletin des postes vacants de l'Esid sur quatre années de référence, 1999. Consultado el: 26-12-00.

http://www.geneve.ch/heg/id/presentation/missions/urtexte2.htm

Euro-Chambres. FutureProf: Flexible training profiles/curricula meeting the demands of the future, taking account of key qualifications required in the ITC fields of multimedia/EDP, telecommunication, marketing/advertising, 1999. Consultado el 31-08-00.

http://www.webb.co.at/futureprof/summary.html

Fernández, Paz. "Certificación de profesionales en información y documentación: un proceso abierto en España y en la Unión Europea". En: El profesional de la información, 2002, mayo-junio, v. 11, n. 3, pp. 190-194. http://www.sedic.es/artcert.pdf

FID. The current situation: a summary of results from FID's survey of the information professionals. Consultado el 17-08-00.

http://fid.conicyt.cl:8000/mipindex.htm

Gellman, R. "Disintermediation and the internet". En: Government information quarterly, 1996, v. 13, n. 1, pp. 1-8.

Gorman, G. E. "The future of library science education". En: Libri, 1999, v. 49, pp. 1-10.

Institute of Information Scientists. Criteria for information science. Consultado el: 17-08-00.

http://www.iis.org.uk/Criteria.html

Line, M. B. "Le métier de bibliothécaire: un ensemble de pratiques confuses et discontinues". En: Bulletin des bibliothèques de France, 1998, v. 43, n. 2, pp. 44-48. Consultado el: 09-08-00. http://www.enssib.fr/bbf/

Marcial, N. A. "Competencias en el uso de la tecnología y los recursos de información". En: Transinformação, 1999, setembro/dezembro, v. 11, n. 3, pp. 195-204.

Meyriat, J. "Qualifications et certification des professionnels de l'I+D dans huit pays européens: brève présentation comparative". En: Documentaliste-sciences de l'information, 1999, v. 36, n. 2, pp. 113-116.

Meyriat, J. "El profesional de la información en Europa. El proyecto Decidoc. En: X Jornadas de la eubd: profesionales de la información y documentación: formación, perfiles y mercado de trabajo en un entorno europeo, 2001.

New Jersey Library Association. Core competencies for librarians, 1999. Consultado el: 04-02-01.

http://www.njla.org/statements/competencies.html

Noble, C. "Reflecting on our future". En: Computers in libraries, 1998, February, v. 18, n. 2. Consultado el: 20-10-02.

http://www.infotoday.com/cilmag/feb98/story2.htm

Portela, P. "La certificación de profesionales de la información y documentación: experiencias internacionales y propuesta española”. En: Revista española de documentación científica, 1997, octubre-diciembre, v. 20 , n. 4 , pp. 437-449.
Relación de eurocompetencias en información y documentación. Madrid: Sedic, 2000.

Rial Sánchez, A. "La formación para el trabajo: nuevos escenarios, nuevos requerimientos de competencias y cualificaciones". En: Monchís, A. (coord.). Formación y empleo: enseñanza y competencias. Granada: Comares, 2000, pp. 233-255.

Roederer, B. L'évolution de la fonction information-documentation: résultats de l'enquête Adbs 1999. Paris: Adbs, 2000. Isbn 2-84365-038-0.

Scott, P. J.; Kirby, A. E. "The SLA competencies: raising the bar on performance". En: Library journal, 1999, July, v. 124, n. 12, p. 46.

Sebastià Salat, M. La tecnologia de la informació com a àrea disciplinar en el disseny del currículum de formació de professionals i d'usuaris de la documentació $i$ de la informació. Tesis doctoral. Barcelona: Universitat, 1996.

Special Committee on Competencies for Special Librarians, Special Library Association. Competencies for Special Librarians of the 21st Century. 1996. Consultado el: 03-02-01.

http://www.sla.org/professional/competency.html

Special Libraries Association; Association for Library and Information Science Education; Medical Library Association. Competencies for special librarians of the 21st century: Library and information studies programs surveys: final report. 1998.

Spiegelman, B. M. (ed.). Competencies for special librarians for 21 st century. Washington: Special Libraries Association, 1997. Isbn 0-87111469-0.

Stenson, A.; Raddon, R.; Cramer, D. Skills and competencies in the corporate sector. London: British Library Research and Innovation Centre, 1999. Isbn 0-7123-9749-3.

Sturges, P. "The pursuit of content". En: Education for information, v. 17, n. 3, 1999, pp. 175-185.

Tejada Artigas, C.; Rodríguez Yunta, L. Situación laboral y desarrollo profesional de los socios de Sedic: resultados de la encuesta realizada en el 2001. Madrid: Sedic, 2002. Isbn 84-89456-03-8.

Tejada Artigas, C. Adecuación de los planes de estudio de la diplomatura en biblioteconomía y documentación a las demandas del mercado de trabajo. El caso concreto del nuevo plan de estudios de la Escuela Universitaria de Biblioteconomía y Documentación de la Universidad Complutense de Madrid. Tesis doctoral. Madrid: Universidad Carlos III, 2002.

Tfpl. Skills for knowledge management: a briefing paper, 1999. Consultado el: 07-01-01.

http://www.tfpl.com/areas_of_expertisel_knowledge_management/KM_s kills research/KM skills executive summary/KM skills.pdf http.//www.lic.gov.uk/publications/executivesummäries/kmskills.htm

Thierry, C.; Gouérou, G.; Dalbin, S. "Utiliser le référentiel des compétences et emplois de l'Adbs. Un exemple: définir un 'poste composite' associant des fonctions de documentation et de secrétariat". En: Documentaliste-sciences de l'information, 1997, v. 34. n. 2, pp. 104-108.

Valentim, M. P. (org.); Ponjuan Dante, G. [et al.]. O profissional da informação: formação, perfil e atuação profissional. São Paulo: Polis, 2000. Isbn 85-7228-011-1.

Webber, S. "Competencies for information professionals". En: Bulletin of the American Society for Information Science, 1999, October/November, v. 26, n. 1. Consultado el: 02-11-02

http://www.asis.org/Bulletin/Oct-99/webber.html

Wright, S. "Competencies promote partnerships". En: Information outlo$o k, 1997$, v. 1, pp. 26-27.

Carlos Tejada Artigas, Escuela Universitaria de Biblioteconomía y Documentación de la Universidad Complutense de Madrid.

tejada@caelo.eubd.ucm.es

Luis Rodríguez Yunta, miembro de la Junta Directiva de Sedic.

luisry@cindoc.csic.es 